\title{
Effectiveness for Securing Sensitive Data and Supporting Multi Data Applications in Cloud Forensics Environment
}

\begin{abstract}
Distributed computing turned into the most discussed imaginative innovation in Information and correspondence innovation in current patterns. The significant worry of the various associations currently is the security of a client subtleties or archives. In computerized legal examinations and log observing is by and by a significant and open concern. The effect is that patient's subtleties doesn't work be really handled and dissipated nature. The vast majority of the applications need to associate with a few different information stockpiling process contingent upon the sort of information they need to keep up: existing information types, result report, factual information from interpersonal organizations and so on. The significance of utilizing provenance information is critical it gives the criminological agents information history as far as patients, substances and exercises engaged with creating related patients wellbeing information reports. It is difficult to find the client's information where expected to be put away. In this paper, propose a lot of techniques and encryption plot is targeting lightening designers task for creating, sending and moving different information stockpiling progressively assignments and furthermore to upgrade a protected provenance conspire dependent on cutting edge VM measurable computerized assessment investigation apparatuses and square chain innovation. It oversees and screen the internal area of information, both where it originates from as it goes through the information provenance by means of hypervisor.

Watchwords Cloud Forensics, VM legal advanced assessment investigation devices, Block Chain Technology and cryptography plans.
\end{abstract}

Sonya A, Kavitha G

\section{INTRODUCTION}

Distributed computing offers such a large number of advantages for ongoing applications and furthermore different business associations by giving exceptionally high adaptable foundation assets, pay by use strategy and least cost on-request processing [1]. The subject of this idea is to look at how the patients history can be distinguish, examinations and afterward keep up, it very well may be utilized in the cloud so as to follow the past records of the patient's data reports has gone in the cloud. Be that as it may, the primary security risk of distributed computing has become an expanding tension. Malignant people can without much of a stretch take authority capacity to hack the client's or patients delicate subtleties in cloud. A significant number of these assaults are various structures like undesirable messages (spam message), promotions and undesirable sites. Cloud computerized criminology devices can be characterized as an applied science for the ID, conservation, examination and introduction of information while saving the unwavering quality of the patient's data records and keeping up a demanding grouping of insurance for the client's information.

Revised Manuscript Received on October 22, 2019.

Sonya A, Computer science Engineering BSA Crescent institute of science and technology, Chennai-India

Kavitha G, Computer science Engineering BSA Crescent institute of science and technology,Chennai-India
Be that as it may, cloud crime scene investigation likewise incorporates exploring whole patient's document frameworks, execution stream and history. It is hard to envision the emergency clinic the board framework can spare all its patient private information and keep the security assurance for that data. When the patients get check up, need not to be check over and over. The patient's subtleties can get to legitimately through their cloud framework. It prompts time utilization. It can get to just by utilizing OTP or Password in other framework. Virtual cloud can be introducing when the power is shutdown or no electricity.[2] Thus, the idea of this discourse is to destruction and decreasing the constant issues in cloud and recognizes a proper arrangement that can be helpful in up and coming innovation with ideal use asset to store the patients subtleties with a verified way. [3] It coordinates various existent and new advances, for example, equipment virtualization, versatile virtualization, work area virtualization, and so forth and concentrated registering. It gives perpetual 'mind boggling' sources to clients as administrations over the Internet which supportive to get the subtleties from patients. [4]A human services investigation reports are required to contain provenance subtleties on the pathway the information took during different phases of preparing of different undertakings. In this manner, give provenance to keep up the possession and procedure history of patients information traits in the information provenance store. We have to give insurance and security for patient's general information and furthermore private information. The necessities incorporate honesty of archives, enforceability of the provenance record, and confirmation of the client's character. The primary objective is to propose a viable secure provenance plot with Hash Functions which can give VM log checking to information crime scene investigation in cloud legal sciences. The information provenance records will give computerized specialists a reasonable record of where the information has been travel in the cloud legal sciences.

It likewise gives uprightness on delicate records put away in a cloud. In the event that somebody like a programmer or unapproved outsiders attempting to bargains information trustworthiness or privacy of such a problematic occurrence, the nature of work for legal sciences groups is to research at that point report and update the data. Our primary idea is capable misuse in cloud criminology for getting wanted point of discovery and avoidance of fabrication and offenders.

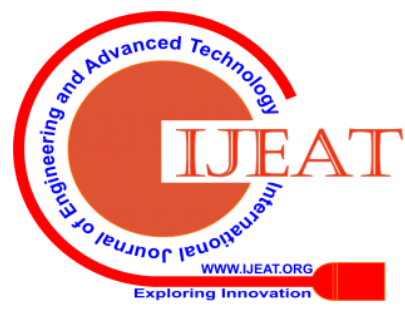




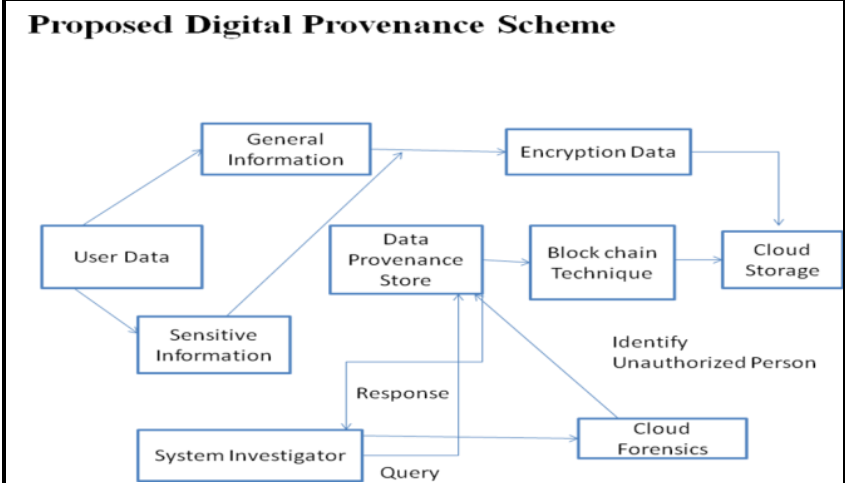

Fig 1. Diagrammatical way of proposed system

In the proposed plan, client information are classified into two different ways like general data and delicate data. Both general data and touchy data are scrambled by hash capacities put away in the distributed storage. It deals with the information provenance store. It gives the help to putting away the source information relating to the information provenance. The source information is put away in the Data Provenance Store which is additionally overseen by a CSP. While both the User information and the framework agents are in the confided in condition. A client information has taken a contribution of source information, sends the relating information provenance to the Provenance Store. A framework specialist may (1) send a solicitation to the Provenance Store and subsequently he/she (2) gets the affirmation. Assume if the framework examiner recognizes the unapproved client attempting to follow the information. It takes the computerized provenance utilizing crime scene investigation procedure to locate the unapproved client. At last Data crime scene investigation is put away in information provenance stockpiling. At the point when an assortment of various patients' subtleties is approved to produce reports, if some issue emerges in an alteration, the concerned patient can be recognized by some assigned gathering utilizing crime scene investigation examination utilizing square chain.

\section{A. methodology}

\section{1) Block Chain Technology}

A square chain is an expanding chain of reports called squares, which are affixed utilizing cryptography. Data hung on a square chain exists as a conveyed and constantly subset in database. This innovation database need not to be accessible in any single site, that implies every one of the reports keeps are generally open and effectively auditable and obvious. Here there is no unified report of this data exists for a programmer to abnormal. Facilitated by a great many PCs simultaneously, its data is available to anybody at wherever by utilizing web. It ensures our touchy information, report and records, both on the hubs and in transmission, by utilizing square chain innovation and cryptography to encode the documents. This framework is pictured where each part of distributed storage, for example, transmission, producing or information stockpiling is gone into the square chain. After this, which data ought to be investigated, where it went, who got to the data, and how that data was observed can be checked by any individual who has authority capacity to create to the square chain. Basically, the square chain fixed the processing stage in time. It helps in giving total recognizable, duty, and understandability for the cloud.All clients and client's touchy information in disseminated distributed storage are interlinked organize. It gives a lot of dependable, quicker, and more affordable than the current frameworks. Along these lines, distributed storage empowers clients to store client's touchy information in a safe way. It is finished by utilizing square chain highlights, for example, exchange records, hash capacities, and cryptographic key encryption. Server farms are the center point of distributed storage capacities for cloud monsters like many open sources. Be that as it may, these server farms are advertising with a significant expense tag for cloud engineers, suppliers, and clients. And furthermore the effect of this greater expense of information disappointments and security breaks. The square chain is upsetting distributed storage by returning the client responsible for their information and gadgets. One reason square chain is considered, in light of the fact that it is straightforwardness to all with troublesome way. Anybody can access to the web, it will check the chain and see for them in the case of everything up till now is reasonable. Another explanation is a direct result of the evidence of-work idea square chain work. Coming up next are the three significant advantages of circulated distributed storage.

\section{2) Tamper-confirmation information}

Reinforcement and capacity specialists have demonstrated that spare client information has not been messed with, when a viable and evident reinforcement was presented and made. Circulated distributed storage dependent on square chain innovation spare just hashes of its information obstructs for clients touchy information. What's more, the encoded and disseminated hashes are essential to check these client information squares.

\section{3) Verifiability}

Square chain is simply not just spare information in an appropriated and encoded group, yet additionally accommodates direct chain in which each square contains a cryptographic hash capacities. This connects to the squares and along these lines, makes a decentralized exchange record.

\section{4) No more mediators}

Many cloud specialists, the greatest effect that square affixes are probably going to bring is to serve the item legitimately to the client. This is on the grounds that a well-planned and openly available square chain can supplant .It for the most part giving a reliable exchanging condition, guarding against extortion and dealing with, guaranteeing contract consistence, and monetary exchanges the board framework. Square chain's capacity doesn't lie in its troublesome way; its conveyance over a chain of PCs likewise makes square affix more diligently to assault. This innovation is available by possess self confirming consecutive stockpiling schme.

\section{A. modules descripition}

- Attributes definition/Master Secret key generation module

- Access structure Specification Module

- Private key generation module

- Transformation Key generation Module

- Retrieving Key Generation Module

- Final performance evaluation module

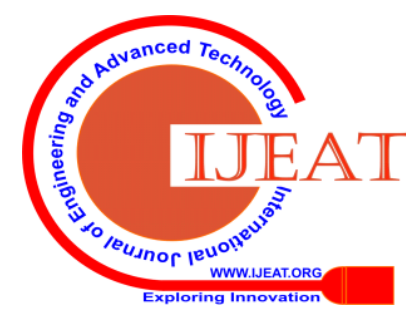


a) Attributes definition/master secret key

- An attribute is a one kind of data which access the properties of a field or tag in a database or a string of characters in a display.

- A key is considered as numeric that contains the output of an encryption algorithm when the condition is exchange from plain text to cipher text.

- Master secret key is a cryptographic key used in protected data communication.

- In this module, a master secret key is generated from the inputs provided by the user and the finalisation of attributes to be used for encryption.

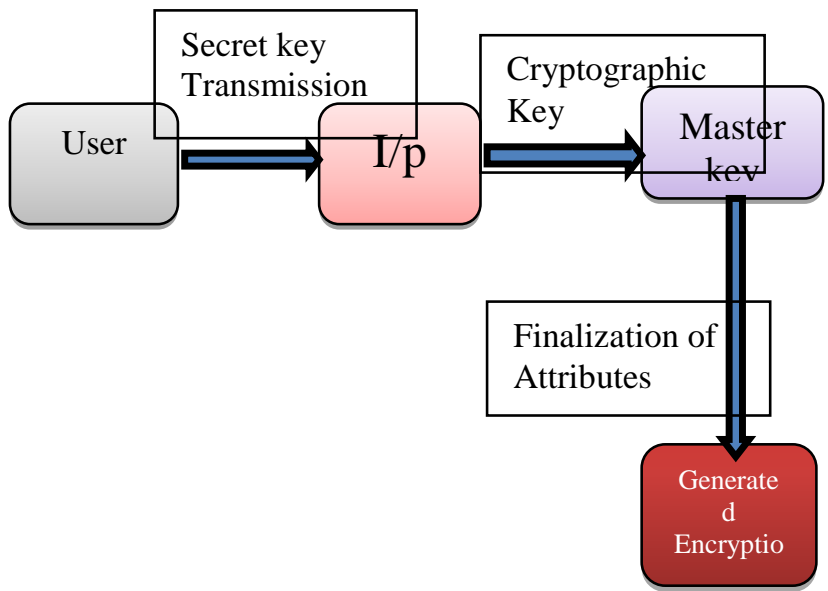

Fig 2. Attributes definition /Master secret key

b) Access structures specification module

- A detailed description of the design and materials used to make something is specification. Access structures are used to study the structure of security system. So where multiple workers need to work together to obtain a necessary source.

- Based on the access structure of the users the data will be encrypted for security purpose.

- So that, the algorithm will be defined dynamically for data encryption.

The message to be passed is converted into cipher text.

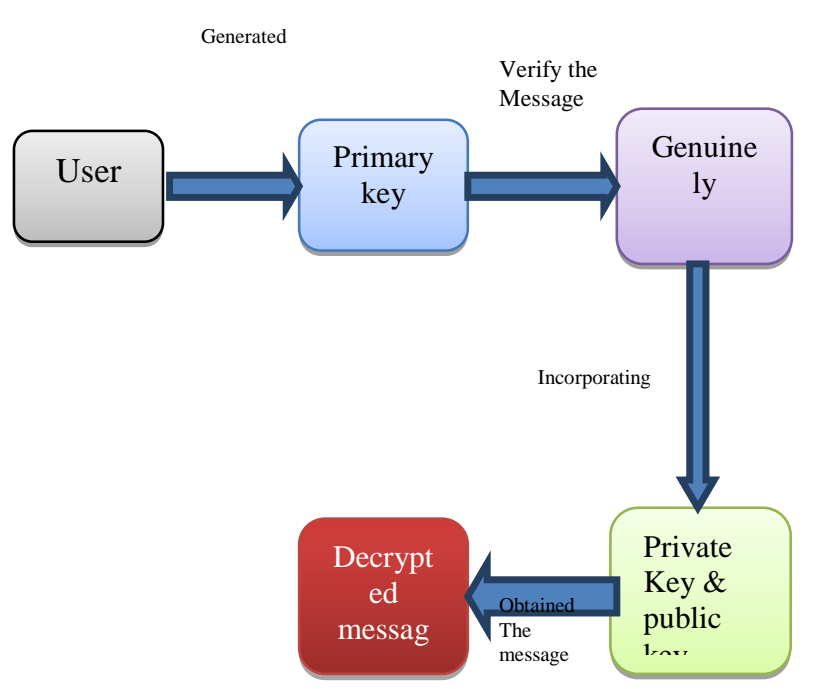

Fig 4. Private Key generation module

\section{c) Transformation key generation module}

$>$ A Partialised algorithmic approach is used in this module so as to decrypt the cipher text into partially decrypted cipher text.

$>$ The final data can be generated from the partially ciphered text.

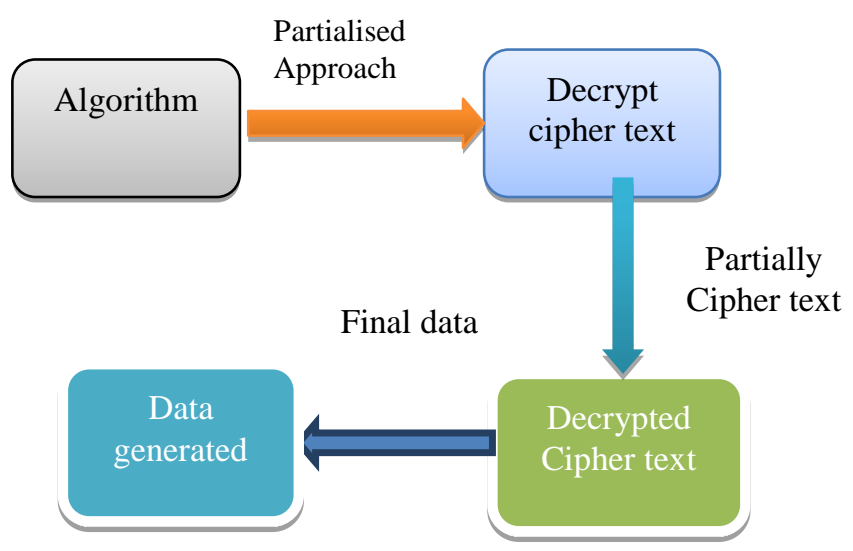

Fig 5. Transformation key generation

d) Retrieve key generation module

- Retrieve is defined as to find and bring or to bring back or restore, whether physically or mentally. The process of decoding data that has been encoded into a secured format.

- Retrieving key is a cryptographic key used to retrieve the partial decrypted information fully.

- In this module, Partial key can be decrypted further to receive the final message which is validated and compared with the decrypted one received in the third module.

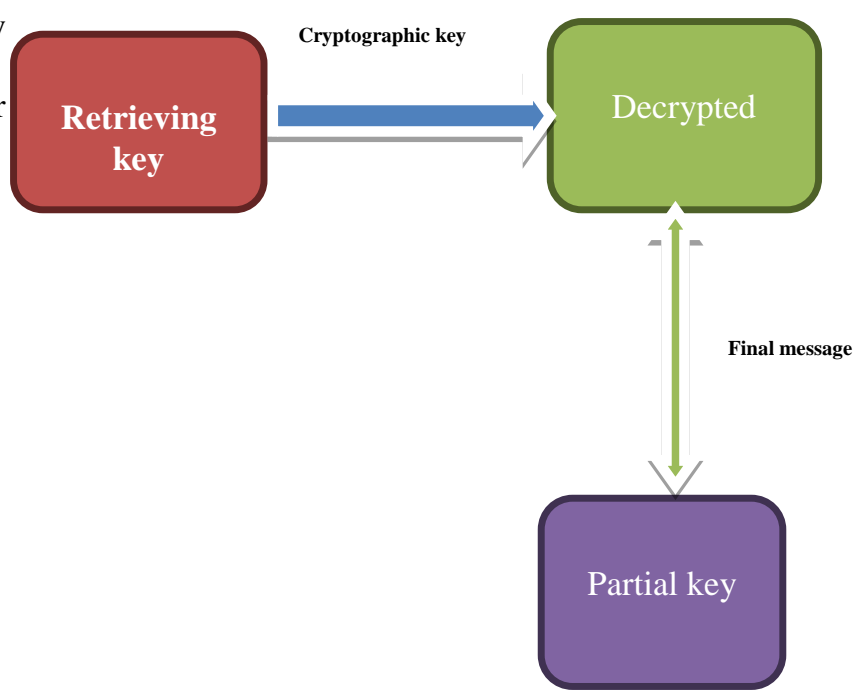

Fig 6.Retrieve key generation module 


\section{Effectiveness for Securing Sensitive Data and Supporting Multi Data Applications in Cloud Forensics Environment}

\section{CONCLUSION}

Hence the objective considered thereby defining and verifying a patient document to check the patient details like attributes and master secret key using block chain technology depending on encryption and decryption techniques. It will achieve much private and fine-grained data access control, proficient and integrity the user data with a secure manner. In this paper, propose a set of methods and encryption scheme is aiming at alleviating developers task for developing, deploying and migrating multiple data storage in real time tasks and also to enhance a secure provenance scheme based on advanced VM forensic digital examination analysis tools and block chain technology. It helps to manage and monitor the inner location of data, both where it comes from as it passes through the data provenance via hypervisor.

\section{REFERENCES}

1. B. Waters, "Ciphertext-policy attribute-based encryption: An expressive, efficient, and provably secure realization,” in Proc. Public Key Cryptography, 2012, pp. 53-70.

2. NuttapongAttrapadung, Javier Herranz, Fabien Laguillauimie, Benoit Libert, Elie de Panafieu, and Carla Rafols. Attribute-based encryption schemes with constant-size ciphertexts. Theor. Comput. Sci., 422:1538, 2012.

3. J Ayo Akinyele, Gary Belvin, Christina Garman, Matthew Pagano, Michael Rushanan, Paul Martin, Ian Miers, Matthew Green, and Avi Rubin. Charm: A tool for rapid cryptographic prototyping. Available from http://www.charm-crypto.com/, 2012.

4. "State of blockchain q1 2016: Block chain funding overtakes bitcoin," 2016. [Online]. Available: http://www.coindesk.com/ state-ofblockchain-q1-2016/

5. Kosba, A. Miller, E. Shi, Z. Wen, and C. Papamanthou, "Hawk: The block chain model of cryptography and privacy-preserving smart contracts," in Proceedings of IEEE Symposium on Security and Privacy (SP), San Jose, CA, USA, 2016, pp. 839-858.

6. Kan Yang and Xiaohua Jia," An Efficient and Secure Dynamic Auditing Protocol for Data Storage in Cloud Computing" in the IEEE Transactions on Parallel and Distributed System, Vol. 24, No. 9 , September -2013.

7. S.Hemalatha and S.Alaudeen Basha,"Enabling for Cost-Effective Privacy Preserving of Intermediate Data Sets in Cloud" Journal of Scientific and Research Publications,vol.3 issue.10,oct 2013.

8. C.Celcia and T.Kavitha,"Privacy Preserving Heuristic Approach for Intermediate Data Sets in Cloud", Journal of Engineering Trends and Technology, vol.9, no.5, march 2014.

9. A.Sonya' Dr. G.Kavitha' Efficient approaches for storing retrieval and replication of data using cloud system. International Journal of Pure and Applied Mathematics Volume 117 No. 20 2017, 1011-1020 ISSN: 1311-8080 ISSN: 1314-3395 Nov 17.

10. Dineshkumar C, Subramanian M "Experimental Investigation of Onboard Driver Condition Monitoring System for Passenger Vehicles" International Journal of Mechanical Engineering and Technology (IJMET) Volume9, Issue6, June 2018, pp.01-09. Article ID: IJMET_06_07_001.

11. A.Sonya Clustering of Crime Information by Using Mobile Application, International Journal of Engineering Research \& Management Technology September-2018 ISSN: 2348-4039 Volume 5 , Issue- 5 . 